\title{
HOMOTOPY FOR CELLULAR SET-VALUED FUNCTIONS
}

T. R. BRAHANA, ${ }^{1}$ M. K. FORT, JR., ${ }^{2}$ AND WALT G. HORSTMAN ${ }^{3}$

1. Introduction. A. Granas asked the following question. If $F$ is an upper semi-continuous set-valued function on a compact metric space $M$ such that the image of each point of $M$ is a proper subcontinuum of $S^{n}$, then is $F$ "homotopic" to a single-valued continuous function? It was pointed out that care must be used in the definition of homotopy of set-valued functions, since the first natural candidate puts all upper semi-continuous set-valued functions into one class. In [3] and [2] studies were made of homotopies of set-valued functions subject to the restriction that $H(x, t)$ be acyclic (with respect to homology over $\left.Z_{2}\right)$ for each $(x, t) \in M \times I$.

In this paper the homotopy problem is solved for those upper semicontinuous functions $F$ for which each $F(x)$ is a cellular subset of $S^{n}$. In particular, the class of cellular upper semi-continuous set-valued functions is partitioned into equivalence classes by the relation of cellular homotopy, each class contains single-valued continuous functions, and two single-valued continuous functions are in the same class if and only if they are homotopic in the usual sense.

A selection theorem which seems to be different from those discussed in the literature is proved in $\$ 2$. It is shown that if $F$ is upper semi-continuous on $M$ and $F(x)$ is a cellular subset of $S^{n}$ for each $x \in M$, then there exists a continuous function $g: M \rightarrow S^{n}$ such that $g(x) \in S^{n}-F(x)$ for each $x \in M$. In addition to being the main tool used in the construction of the homotopies, this selection theorem is of interest in itself.

2. The selection theorem. A subset $A$ of $S^{n}$ is cellular if and only if there exists a sequence $E_{1} \supset E_{2} \supset E_{3} \supset \cdots$ of topological $n$-cells such that $A=\bigcap_{k=1}^{\infty} E_{k}$ and, for each $k, A \subset$ interior $E_{k}$.

Let $M$ be an $m$-dimensional compact metric space and let $F$ be a set-valued function on $M$ such that:

(i) for each $x \in M, F(x)$ is a cellular subset of $S^{n}$, and

(ii) $F$ is upper semi-continuous.

A covering pair for $F$ and $M$ is an ordered pair $(G, D)$ such that:

Received by the editors December 23, 1963.

1 Supported by the National Science Foundation research grant G-10096.

2 Professor Fort died on August 2, 1964. Research had been supported by the National Science Foundation research grant G-23790.

${ }^{3}$ Supported by the National Science Foundation research grant G-10096. 
(i) $G$ is a finite open covering of $M$,

(ii) $D$ is a function with domain $G$ such that for each $U \in G, D(U)$ is a topological $n$-cell which is contained in $S^{n}$, and

(iii) for each $x \in M$, if $x \in U \in G$ then $F(x)$ Cinterior $D(U)$.

LEмma 1. There exists a covering pair.

Proof. For each $x \in M$, there exists a topological $n$-cell $\Delta(x)$ such that $F(x) \subset$ interior $\Delta(x)$ and $\Delta(x) \subset S^{n}$. For each $x \in M$, there exists a neighborhood $V(x)$ such that if $t \in V(x)$ then $F(t) \subset$ interior $\Delta(x)$. $\{V(x) \mid x \in M\}$ is an open covering of $M$, and, since $M$ is compact, this covering has a finite subcovering $G=\left\{V\left(x_{1}\right), \cdots, V\left(x_{k}\right)\right\}$. We define $D\left(V\left(x_{j}\right)\right)=\Delta\left(x_{j}\right)$ for $j=1, \cdots, k$. It is easy to verify that $(G, D)$ is a covering pair.

LEMma 2. If $(G, D)$ is a covering pair, then there exists a covering pair $\left(G^{*}, D^{*}\right)$ such that:

(i) $G^{*}$ is a star refinement of $G$, and

(ii) if $U \in G, U^{*} \in G^{*}$ and $U^{*} \subset U$, then $D^{*}\left(U^{*}\right) \subset D(U)$.

Proof. Let $\lambda$ be the Lebesgue number of the covering $G$. For each $x \in M$, there is a topological $n$-cell $\Delta(x)$ such that $F(x) \subset$ interior $\Delta(x)$ and such that if $x \in U \in G$ then $\Delta(x) \subset D(U)$. For each $x \in M$, there is a neighborhood $W(x)$ of $x$ such that:

(i) $W(x)$ is contained in the $\lambda / 3$-neighborhood of $x$, and

(ii) if $t \in W(x)$ then $F(t) \subset$ interior $\Delta(x)$. The set $\{W(x) \mid x \in M\}$ is an open covering of $M$ and has a finite subcover $G^{*}=\left\{W\left(x_{1}\right), \cdots\right.$, $\left.W\left(x_{k}\right)\right\}$. We define $D^{*}\left(W\left(x_{j}\right)\right)=\Delta\left(x_{j}\right)$ for $j=1, \cdots, k$. It is easy to verify that $\left(G^{*}, D^{*}\right)$ has the desired properties.

Let $F$ be an upper semi-continuous set-valued function on a compact finite-dimensional metric space $M$ such that, for each $x \in M$, $F(x)$ is a cellular subset of $S^{n}$.

Theorem 1. There exists a single-valued continuous function $g: M \rightarrow S^{n}$ such that $g(x) \in S^{n}-F(x)$ for each $x \in M$.

Proof. Let $m$ be the dimension of $M$. It follows from Lemmas 1 and 2 that there are covering pairs $\left(G_{0}, D_{0}\right), \cdots,\left(G_{2 m}, D_{2 m}\right)$ such that for each $j, 1 \leqq j \leqq 2 m$ : (i) $G_{j}$ is a star refinement of $G_{j-1}$, and (ii) if $U_{j} \in G_{j}, U_{j-1} \in G_{j-1}$ and $U_{j} \subset U_{j-1}$, then $D_{j}\left(U_{j}\right) \subset D_{j-1}\left(U_{j-1}\right)$.

We choose a finite open covering $G$ of $M$ such that $G$ is of order $m$, $G$ is a star refinement of $G_{2 m}$, and no proper subset of $G$ covers $M$. For each integer $j, 0 \leqq j \leqq m$, we define $K_{j}=\{x \mid x \in M$ and $x$ is a member of at most $j+1$ members of $G\}$. Each $K_{j}$ is a closed subset of $M$, and $K_{m}=M$. 
For each $V \in G$ and each integer $j, 0 \leqq j \leqq 2 m$, we select sets $\phi_{j}(V)$ $\in G_{j}$ such that $\operatorname{St}(V) \subset \phi_{2 m}(V)$ and $\operatorname{St}\left(\phi_{j}(V)\right) \subset \phi_{j-1}(V)$, for $1 \leqq j \leqq 2 m$. We define $\Phi_{j}(V)=D_{j}\left(\phi_{j}(V)\right)$ for each $V \in G$ and $j=0, \cdots, 2 m$.

We are going to define (inductively) for each $j, 0 \leqq j \leqq m$, a mapping $g_{j}: K_{j} \rightarrow S^{n}$ such that, for each $V \in G$,

$$
g_{j}\left[V \cap K_{j}\right] \subset \mathrm{Cl}\left[S^{n}-\Phi_{2 j}(V)\right] .
$$

For each $V \in G$, we choose a point $p_{V} \in S^{n}-\Phi_{0}(V)$ and define $g_{0}(x)$ $=p_{V}$ for each $x \in V \cap K_{0}$. Since $V \cap K_{0}$ is closed in $K_{0}$ for each $V \in G$, $g_{0}$ is continuous.

Now suppose $0<j \leqq m$ and $g_{j-1}$ has been defined. Let $\sigma=\left\{V_{0}, \cdots, V_{j}\right\}$ be a set of $j+1$ distinct members of $G$ such that $V_{0} \cap \cdots \cap V_{j} \neq \varnothing$. We define $H_{\sigma}=\left(V_{0} \cap \cdots \cap V_{j}\right) \cap K_{j}$ and $W_{0}$ $=K_{j}-\cup\{V \mid V \in G-\sigma\}$. Then $W_{\sigma}$ is closed in $K_{j}$ and $H_{\sigma}$ is open relative to $W_{\sigma}$. The mapping $g_{j-1}$ is defined on $W_{\sigma}-H_{\sigma}$ and $W_{\sigma}-H_{\sigma}$ is closed relative to $W_{\sigma}$. It is easy to see that

$$
\begin{aligned}
g_{j-1}\left[W_{\sigma}-H_{\sigma}\right] & \subset \bigcup_{r=0}^{j} \mathrm{Cl}\left[S^{n}-\Phi_{2 j-2}\left(V_{r}\right)\right] \\
& =\mathrm{Cl}\left[S^{n}-\bigcap_{r=0}^{j} \Phi_{2 j-2}\left(V_{r}\right)\right] .
\end{aligned}
$$

Since $\phi_{2 j-1}(V) \subset \operatorname{St}\left(\phi_{2 j-1}\left(V_{r}\right)\right) \subset \phi_{2 j-2}\left(V_{r}\right)$ for $r=0, \cdots, j, \Phi_{2 j-1}\left(V_{0}\right)$ $\subset \cap_{r=0}^{j} \Phi_{2 j-2}\left(V_{r}\right)$. Therefore, $g_{j-1}\left[W_{\sigma}-H_{\sigma}\right] \subset \mathrm{Cl}\left[S^{n}-\Phi_{2 j-1}\left(V_{0}\right)\right]$.

The set $\mathrm{Cl}\left[S^{n}-\Phi_{2 j-1}\left(V_{0}\right)\right]$ is the union of a topological $(n-1)$ sphere $\Sigma$ and one of the components of $S^{n}-\Sigma$. It is known (see [1]) that such sets are absolute retracts. Since $\mathrm{Cl}\left[S^{n}-\Phi_{2 j-1}\left(V_{0}\right)\right]$ is an absolute retract, we can extend $g_{j-1} \mid\left(W_{\sigma}-H_{\sigma}\right)$ to a mapping

$$
\psi_{\sigma}: W_{\sigma} \rightarrow \mathrm{Cl}\left[S^{n}-\Phi_{2 j-1}\left(V_{0}\right)\right] \text {. }
$$

Since $V_{r} \subset \operatorname{St}\left(V_{0}\right)$ for $r=0, \cdots, j$,

$$
\phi_{2 j}\left(V_{r}\right) \subset \operatorname{St}\left(\phi_{2 j}\left(V_{0}\right)\right) \subset \phi_{2 j-1}\left(V_{0}\right) .
$$

Thus $\Phi_{2 j}\left(V_{r}\right) \subset \Phi_{2 j-1}\left(V_{0}\right)$ and the range of $\psi_{\sigma}$ is contained in $\mathrm{Cl}\left[S^{n}-\Phi_{2 j}\left(V_{r}\right)\right]$ for $r=0, \cdots, j$. Thus $\psi_{\sigma}\left[V_{r} \cap W_{\sigma}\right] \subset \mathrm{Cl}\left[S^{n}-\Phi_{2 j}\left(V_{r}\right)\right]$ for $r=0, \cdots, j$.

If $\sigma^{\prime}$ is a different system of $j+1$ members of $G$ and $W_{\sigma^{\prime}} \cap W_{\sigma} \neq \varnothing$, then $\psi_{\sigma}\left|\left(W_{\sigma^{\prime}} \cap W_{\sigma}\right)=\psi_{\sigma^{\prime}}\right|\left(W_{\sigma^{\prime}} \cap W_{\sigma}\right)=g_{j-1} \mid\left(W_{\sigma^{\prime}} \cap W_{\sigma}\right)$. It follows that we can piece the mappings $\psi_{\sigma}$ and $g_{j-1}$ together to obtain a mapping $g_{j}: K_{j} \rightarrow S^{n}$. It is obvious that $g_{j}\left[V \cap K_{j}\right] \subset \mathrm{Cl}\left[S^{n}-\Phi_{2 j}(V)\right]$ for each $V \in G$.

We define $g=g_{m}$. Since $K_{m}=M, g$ is a mapping of $M$ into $S^{n}$ such 
that $g[V] \subset \mathrm{Cl}\left[S^{n}-\Phi_{2 m}(V)\right]$ for each $V \in G$. If $x \in V \in G$, then $x \in \phi_{2 m}(V)$ and, hence, $F(x) \subset$ interior $\Phi_{2 m}(V)$. It follows that if $x \in M$, then $g(x) \in S^{n}-F(x)$.

3. Homotopy for a class of set-valued functions. Let $M$ be a finitedimensional compact metric space. We define $\Gamma\left(M, S^{n}\right)$ to be the set of all upper semi-continuous set-valued functions $F$ on $M$ such that for each $x \in M, F(x)$ is a cellular subset of $S^{n}$. We let $I=[0,1]$.

Let $F$ and $G$ be members of $\Gamma\left(M, S^{n}\right)$. A function $H$ is a cellular homotopy relating $F$ to $G$ if:

(i) $H \in \Gamma\left(M \times I, S^{n}\right)$, and

(ii) for all $x \in M, H(x, 0)=F(x)$ and $H(x, 1)=G(x)$.

If there exists a cellular homotopy relating $F$ to $G$, then we say that $F$ is homotopic to $G$ and write $F \sim G$. The relation $\sim$ is an equivalence relation and partitions $\Gamma\left(M, S^{n}\right)$ into equivalence classes which we call cellular homotopy classes.

Let $F \in \Gamma\left(M, S^{n}\right)$ and let $f: M \rightarrow S^{n}$ be a (single-valued) continuous function. A function $H$ is a special homotopy relating $F$ to $f$ if :

(i) $H$ is a cellular homotopy relating $F$ to $f$, and

(ii) for all $x \in M$ and $0 \leqq t<1, H(x, t)$ is homeomorphic to $F(x)$.

If $F$ is a single-valued function as well as $f$, then (ii) implies that $H$ is single-valued, and since upper semi-continuity is equivalent to continuity for single-valued functions, in this case $H$ is an ordinary homotopy.

Lemma 3. If $F \in \Gamma\left(M, S^{n}\right)$, then there exists a single-valued continuous function $f: M \rightarrow S^{n}$ and a special homotopy $H$ relating $F$ to $f$. by

Proof. For each $p \in S^{n}$, we define a mapping $J_{p}:\left[S^{n}-p\right] \times I \rightarrow S^{n}$

$$
J_{p}(x, t)=[-t p+(1-t) x] /\|-t p+(1-t) x\|
$$

for $x \in S^{n}-p, 0 \leqq t \leqq 1 . J_{p}$ is a pseudo-isotopy, since the map $\phi_{t}$ defined by $\phi_{t}(x)=J_{p}(x, t)$ is a homeomorphism on $S^{n}-p$ if $0 \leqq t<1, \phi_{0}$ is the identity mapping on $S^{n}-p$, and $\phi_{1}$ is the constant map which takes $S^{n}-p$ into $-p$.

By Theorem 1, there is a mapping $g: M \rightarrow S^{n}$ such that $g(x) \in S^{n}$ $-F(x)$ for each $x \in M$. We define $f(x)=-g(x)$ and

$$
H(x, t)=\left\{J_{\theta(x)}(y, t) \mid y \in F(x)\right\}
$$

for $x \in M$ and $0 \leqq t \leqq 1$. Obviously, $f$ is a continuous function on $M$ into $S^{n}$, and it is easy to verify that $H$ is a special homotopy relating $F$ to $f$. 
THEOREM 2. Each cellular homotopy class of $\Gamma\left(M, S^{n}\right)$ contains a single-valued continuous function $f: M \rightarrow S^{n}$.

PROOF. This result follows immediately from Lemma 3 and the fact that special homotopies are cellular homotopies.

Our final theorem shows that the notion of cellular homotopy which we have defined for $\Gamma\left(M, S^{n}\right)$ is a true extension of the usual notion of homotopy for single-valued functions.

THEOREM 3. If $f_{0}$ and $f_{1}$ are single-valued continuous functions on $M$ into $S^{n}$ and $H \in \Gamma\left(M \times I, S^{n}\right)$ is a cellular homotopy relating $f_{0}$ to $f_{1}$, then there exists a single-valued homotopy $h: M \times I \rightarrow S^{n}$ which relates $f_{0}$ to $f_{1}$ in the usual sense.

Proof. We apply Lemma 3 (replacing $M$ by $M \times I$ and $F$ by $H$ ) to obtain a single-valued continuous function $\phi: M \times I \rightarrow S^{n}$ and a special homotopy $K \in \Gamma\left((M \times I) \times I, S^{n}\right)$ relating $H$ to $\phi$. Now, for $(x, t) \in M \times I$, we define

$$
h(x, t)=\left\{\begin{array}{l}
K(x, 0,3 t) \text { if } 0 \leqq t \leqq 1 / 3 \\
K(x, 3 t-1,1) \text { if } 1 / 3 \leqq t \leqq 2 / 3, \\
K(x, 1,3-3 t) \text { if } 2 / 3 \leqq t \leqq 1
\end{array}\right.
$$

If $0 \leqq t<1 / 3$, then $h(x, t)=K(x, 0,3 t)$ is homeomorphic to $K(x, 0,0)$ $=H(x, 0)=f_{0}(x)$ and, hence, is a one-point set. Likewise, if $2 / 3<t$ $\leqq 1$, then $h(x, t)$ is a one-point set. If $1 / 3 \leqq t \leqq 2 / 3$, then $h(x, t)$ $=K(x, 3 t-1,1)=(x, 3 t-1)$ and since $\phi$ is single-valued, $h(x, t)$ is a one-point set. Thus $h$ is a single-valued function on $M \times I$ into $S^{n}$. Since $K$ is upper semi-continuous, $h$ is also upper semi-continuous. Since $h$ is single-valued, this implies that $h$ is continuous.

We have shown that $h: M \times I \rightarrow S^{n}$ is an ordinary single-valued homotopy. Since $h(x, 0)=K(x, 0,0)=H(x, 0)=f_{0}(x)$ and $h(x, 1)$ $=K(x, 1,0)=H(x, 1)=f_{1}(x), h$ relates $f_{0}$ to $f_{1}$ in the usual sense.

It should be remarked that it can be shown that the smallest equivalence relation containing both homotopies of single-valued functions and special homotopies is the relation generated by cellular homotopies. The proof is similar to that of Theorem 4 .

\section{BIBLIOGRAPHY}

1. R. H. Bing, Retractions onto spheres, Amer. Math. Monthly 71 (1964), 481-484.

2. A. Granas and J. W. Jaworowski, Some theorems on multivalued mappings of subsets of the Euclidean space, Bull. Acad. Polon. Sci. Ser. 7 (1959), 277-283.

3. J. W. Jaworowski, Some consequences of the Vietoris mapping theorem, Fund. Math. 45 (1958), 261-272.

UNIVERSity of Georgia 